\title{
Multiparticle simulation of adiabatic excitation of longitudinal parametric resonances
}

\author{
K. A. Brown, M. Bai, W. Fischer, and T. Roser \\ Brookhaven National Laboratory, Upton, New York 11973 \\ (Received 27 September 2000; published 26 January 2001)
}

\begin{abstract}
In order to further understand phenomena observed during studies of adiabatic excitation of longitudinal bunch shape oscillations [M. Bai et al., Phys. Rev. ST Accel. Beams 3, 064001 (2000)], we have developed a simulation using a one-turn map. In this report we will present the physical foundations for the simulation and the methods used in the simulator. We will present simulation results using parameters of actual experiments, along with the corresponding experimental results.
\end{abstract}

DOI: 10.1103/PhysRevSTAB.4.014001

PACS numbers: 41.85.Ct, 29.27.Bd, 29.27.Fh

\section{INTRODUCTION}

In Ref. [1] it was shown that, by exciting longitudinal bunch shape oscillations adiabatically, longitudinal emittance can be preserved and the driven bunch shape oscillations can be maintained for a long period. To understand the phenomena observed during our studies we developed a single particle dynamics simulation which is able to precisely probe the behavior of particle distributions. In this report we will present data collected over multiple regimes of longitudinal parametric resonances and simulation results corresponding to that data.

The physical foundations for the simulation are given in the discrete synchrotron equations for a stationary bucket, in which the amplitude of the rf voltage is modulated. The synchrotron motion can be described using the conjugate phase space coordinates $\left(\phi, \delta=\frac{h \eta}{\nu_{s}} \frac{\Delta p}{p}\right)$, where $\phi$ is the particle phase relative to the synchronous particle, $h$ is the harmonic number, $\eta=\frac{1}{\gamma_{t}^{2}}-\frac{1}{\gamma^{2}}$ is the phase slip factor, $\nu_{s}$ is the synchrotron tune at zero amplitude without modulation, and $p$ is the particle momentum. The synchrotron tune is given by $\nu_{s}=\sqrt{\frac{h|\eta| e V}{2 \pi \beta^{2} E}}$, with $e$ being the elementary charge, $V$ the gap voltage, $\beta$ the relativistic factor, and $E$ the particle energy. The discrete synchrotron equations for a stationary bucket above transition can then be written as $[2,3]$

$$
\begin{aligned}
\phi_{n+1} & =\phi_{n}+2 \pi \nu_{s} \delta_{n+1}, \\
\delta_{n+1} & =\delta_{n}+2 \pi \nu_{s}\left[1+\epsilon \sin \left(\nu_{m} \theta_{n+1}+\chi\right)\right] \sin \phi_{n},
\end{aligned}
$$

where $\epsilon$ is the modulation percentage of the unperturbed $\mathrm{rf}$ voltage, $\nu_{m}$ is the modulation tune, $\chi$ is the initial modulation phase, and the orbital angle $\theta$ is a timelike variable. Equations (1) correspond to the Hamiltonian

$$
H=\frac{\nu_{s}}{2} \delta^{2}+\nu_{s}\left[1+\epsilon \sin \left(\nu_{m} \theta+\chi\right)\right][1-\cos \phi] .
$$

The canonical transformation for particles within a bucket $\phi=\sqrt{2 J} \cos \psi, \delta=-\sqrt{2 J} \sin \psi$ to action-angle variables $(J, \psi)$ allows going into a rotating coordinate frame. To go into a coordinate system that rotates around the origin with half the modulation tune we use the canonical transformation $J=\tilde{J}, \tilde{\psi}=\psi-\frac{\nu_{m}}{2} \theta-\frac{3 \pi}{4}$. The new Hamiltonian has terms oscillating at $\nu_{m}, 2 \nu_{m}, \ldots$, which average to zero over time, and the time averaged Hamiltonian in this coordinate system is [4]

$$
\langle\tilde{H}\rangle=\left(\nu_{s}-\frac{\nu_{m}}{2}\right) \tilde{J}-\frac{\nu_{s}}{16} \tilde{J}^{2}+\frac{\nu_{s}}{4} \epsilon \tilde{J} \cos 2 \tilde{\psi} .
$$

Since $J=\tilde{J}$ we use $J$ instead of $\tilde{J}$ in the following.

The investigation of fixed points of the Hamiltonian (3) leads to the following result:

(i) If $\nu_{s}\left(2+\frac{\epsilon}{2}\right)<\nu_{m}$, the origin $J=0$ is the only fixed point, a stable one.

(ii) If $\nu_{s}\left(2-\frac{\epsilon}{2}\right)<\nu_{m}<\nu_{s}\left(2+\frac{\epsilon}{2}\right)$, there is one unstable fixed point at the origin and two stable fixed points at $J=8\left(1-\frac{\nu_{m}}{2 \nu_{s}}\right)+2 \epsilon$ and $\tilde{\psi}=0, \pi$.

(iii) If $\nu_{m}<\nu_{s}\left(2-\frac{\epsilon}{2}\right)$, there is a stable fixed point at the origin, two more stable fixed points at $J=8(1-$ $\left.\frac{\nu_{m}}{2 \nu_{s}}\right)+2 \epsilon$ and $\tilde{\psi}=0, \pi$, and two unstable fixed points at $J=8\left(1-\frac{\nu_{m}}{2 \nu_{s}}\right)-2 \epsilon$ and $\tilde{\psi}=\frac{\pi}{2}, \frac{3 \pi}{2}$.

The three cases are illustrated in Fig. 1 where we used the Hamiltonian (3) and the parameters $\nu_{s}=0.0005$ and $\epsilon=0.45$. Figure 1(a1) shows the situation with $\nu_{m}=$ 0.0018 above and far away from the resonance interval $\left[\nu_{s}\left(2-\frac{\epsilon}{2}\right), \nu_{s}\left(2+\frac{\epsilon}{2}\right)\right]$. The phase space trajectories are undisturbed. In Fig. 1(a2) the modulation tune $\nu_{m}=$ 0.0011 is still above but close to the resonance interval. The trajectories in phase space are distorted. Figure 1(b) shows the phase space with $\nu_{m}=0.001$ inside the resonance interval. The unstable fixed point at the origin and the two stable fixed points are clearly visible. In Fig. 1(c1) the modulation tune $\nu_{m}=0.00087$ is below but close to the resonance interval. The appearance of a stable fixed point at the origin and two unstable fixed points above and below it becomes visible. Figure 1(c2) shows the phase space with $\nu_{m}=0.0008$ below and farther away from the resonance interval.

When the amplitude of the modulation is large, the approximations of the Hamiltonian (3) are no longer valid, although it still provides qualitative guidance of the particle 

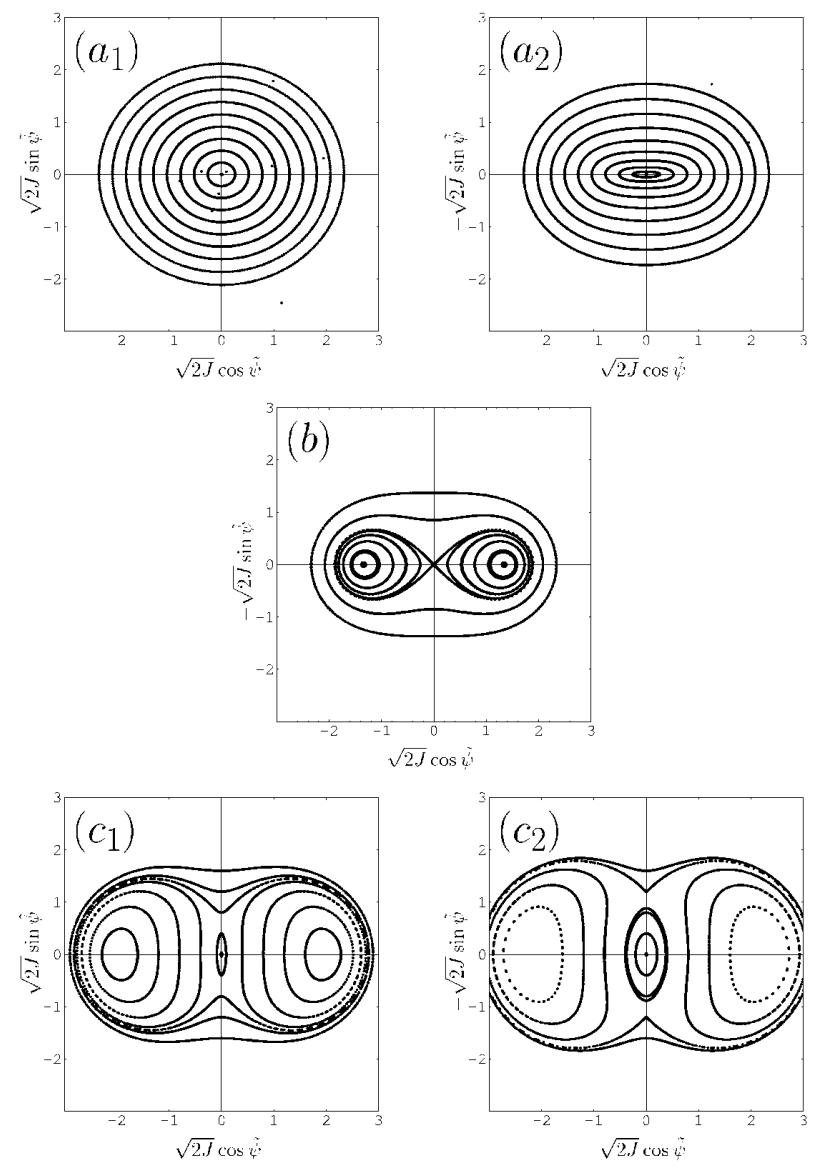

FIG. 1. Simulated particle trajectories in a surface of a section of the Hamiltonian (3) with the parameters $\nu_{s}=0.0005$ and $\epsilon=0.45$. In (a1) the modulation tune $\nu_{m}=0.0018$ is above and far away from the parametric resonance interval, in (a2) it is still above but with $\nu_{m}=0.0011$ close to this interval. (b) shows the situation with $\nu_{m}=0.001$, when the parametric resonance is excited. (c1) and (c2) depict the cases with $\nu_{m}=0.00087$ and $\nu_{m}=0.0008$, respectively, when the modulation tune is below the parametric resonance and either close or far away from that interval.

behavior in phase space. To study more extreme conditions we return to the Hamiltonian (2) and track individual particles.

\section{SIMULATION OF LONGITUDINAL MOTION WITH MODULATED FOCUSING STRENGTH}

We used the one-turn map in Eqs. (1) to simulate the behavior of many particles while incrementing the amplitude slowly over many revolution periods. The simulator did not need to include beam loading effects on the rf voltage since the modulation frequencies fall well within the bandwidth of the Brookhaven alternating gradient synchrotron (AGS) rf automatic gain control loop. With loop gains of $17 \mathrm{~dB}$ and greater the system does a very good job at compensation, reducing to better than $1 \%$ any beam loading distortion $[5,6]$. The simulator did not include space charge effects either, since in the AGS the longitudinal impedances are small. Even at the relatively high intensities and short bunch widths we worked with in our experiments, the space charge effects remain insignificant. To illustrate this, consider the ratio of the induced voltage change $\frac{d V_{z}}{d t}=\frac{Q_{\text {bunch }}}{\sigma_{t}^{3} \sqrt{2 \pi}} \frac{Z_{\|}}{n} \frac{1}{\omega_{0}}$ from space charge to the $\frac{d V_{\text {rf }}}{d t}=V_{\text {rf }} h \omega_{0}$ of the AGS rf system. Given that $\omega_{0}$ is about $2.4 \times 10^{6}, \frac{Z_{\|}}{n}=6 \Omega$ [7] and $V_{\mathrm{rf}} h$ varies from $6 \times$ 20000 to $6 \times 200000$, for a beam intensity of $5 \times 10^{12}$ protons per bunch in a $\sigma_{t}=50 \mathrm{~ns}$ bunch this ratio varies from about $2 \times 10^{-2}$ to $2 \times 10^{-3}$, respectively. From this we conclude that the shift in synchrotron frequency is insignificant $[8,9]$.

Using the parameters of Fig. 1 we mapped out particle trajectories of a surface of a section of the Hamiltonian (2) using the one-turn map in Eqs. (1). The results are shown in Fig. 2. Clearly, the simplified Hamiltonian used to generate the trajectories shown in Fig. 1 is valid only for small amplitude oscillations when the modulation tune is well above twice the synchrotron tune or when the modulation amplitude is small. The trajectories in Fig. 2(c) show that chaotic motion exists in the regions that Fig. 1(c) suggests to be stable regions. Note that the axis labels in Fig. 2 are changed to reflect the canonical phase space variables, $\phi$ and $\delta=\frac{h \eta}{\nu_{s}} \frac{\Delta p}{p}$, as opposed to the rotating frame action angle variables, $\phi=\sqrt{2 J} \cos \tilde{\psi}$ and $\delta=-\sqrt{2 J} \sin \tilde{\psi}$, used in Fig. 1.

\section{COMPARING SIMULATION RESULTS TO EXPERIMENTAL RESULTS}

We operated Brookhaven's AGS with one bunch of a high intensity proton beam at $24 \mathrm{GeV}$, with a harmonic number of 6 and an rms bunch area of $4 \mathrm{eV} \mathrm{s}$. The unmodulated gap voltage seen by the beam was $100 \mathrm{kV}$. These parameters are summarized in Table I.

In Ref. [1] we describe the experimental conditions for our experiments. To summarize, we modulated the AGS rf voltage using a wave form generator that generated a voltage which was added to the gap voltage setting in the low level rf. The combined signal was the input to the rf high level voltage regulation system. In our experiments we slowly increased the parameter $\epsilon$ in Eq. (1) to ensure adiabaticity.

We took four sample cases from our experiments and ran simulations using the same parameters. The four cases are (in all cases $\nu_{s}=0.00022$ and $\epsilon=0.8$ ) (a) the modulation tune is slightly below twice the synchrotron tune, $\nu_{m}=0.000404$. All the beam was lost. (b) The modulation tune is above but close to twice the synchrotron tune, $\nu_{m}=0.000472$. We observed slow beam loss and emittance growth. (c) The modulation tune is above and farther away from twice the synchrotron tune, $\nu_{m}=0.000499$. We observed no beam loss and no emittance growth. 

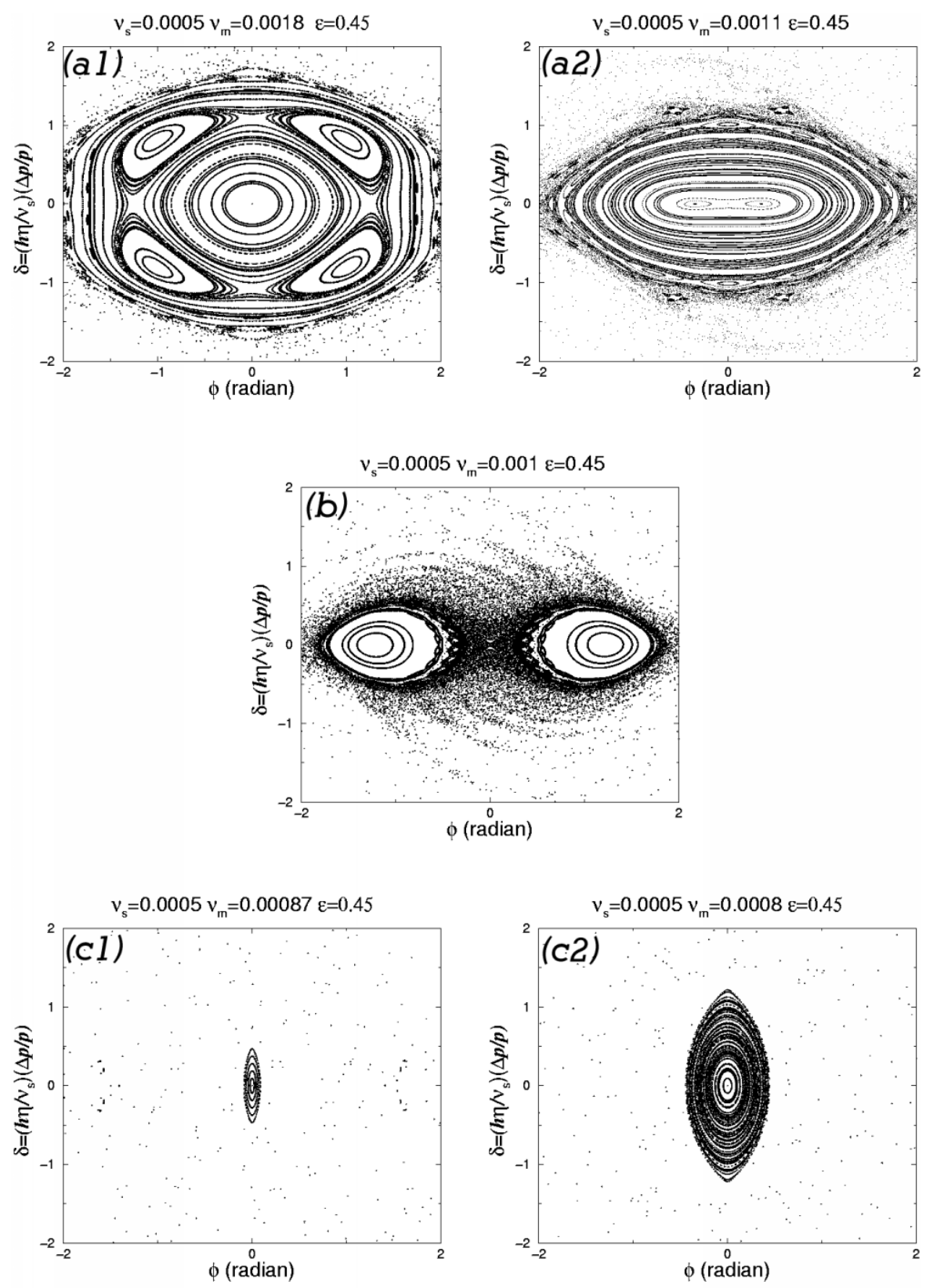

FIG. 2. Simulated particle trajectories in a surface of a section of the Hamiltonian (2) with the same parameters as given in Fig. 1. Note that the axis labels are changed to reflect the canonical phase space variables, $\phi$ and $\delta=\frac{h \eta}{\nu_{s}} \frac{\Delta p}{p}$, as opposed to the rotating frame action angle variables, $\phi=\sqrt{2 J} \cos \tilde{\psi}$ and $\delta=-\sqrt{2 J} \sin \tilde{\psi}$, that were used in Fig. 1.

TABLE I. Basic parameters in the AGS experiment.

\begin{tabular}{lccc}
\hline \hline \multicolumn{1}{c}{ Parameter } & Symbol & Unit & Value \\
\hline Species & $\ldots$ & $\ldots$ & $\mathrm{p}$ \\
Energy & $E$ & $\mathrm{GeV}$ & 24 \\
Harmonic number & $h$ & $\ldots$ & 6 \\
Number of bunches & $\ldots$ & $\ldots$ & 1 \\
Particles per bunch & $\ldots$ & $\ldots$ & $5 \times 10^{12}$ \\
rms bunch area & $\epsilon_{s}$ & $\mathrm{eV} \mathrm{s}$ & 4 \\
Slip factor & $\eta$ & $\ldots$ & 0.0122 \\
Gap voltage & $V_{0}$ & $\mathrm{~V}$ & 100 \\
Synchrotron tune & $\nu_{s}$ & $\cdots$ & 0.00022 \\
\hline \hline
\end{tabular}

(d) The modulation tune is well above twice the synchrotron tune, $\nu_{m}=0.000553$.

Figure 3 shows simulated particle trajectories in the surface of sections of the Hamiltonian (2) for the above four cases. Figure 4 shows results of tracking 50000 particles initially distributed in a Gaussian distribution. The modulation amplitude $\epsilon$ is increased from 0 to 0.8 in ten synchrotron periods. We used the same synchrotron and modulation tunes as in Fig. 3. In Figs. 3(a) and 4(a) we show the phase space before the modulation has reached full amplitude, since particles are lost very quickly. Also note that the scales in Figs. 3(a) and 4(a) show a larger 

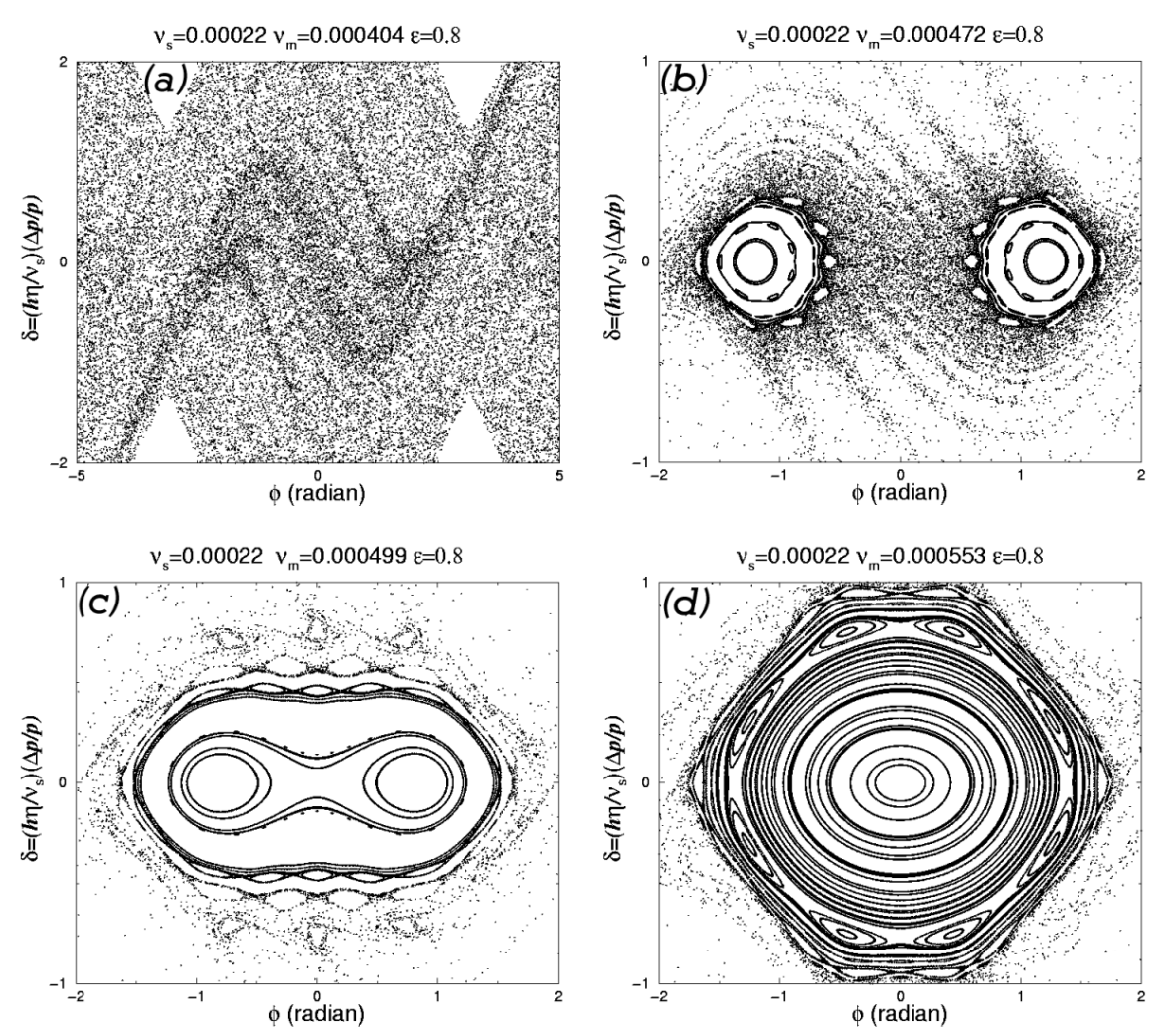

FIG. 3. Simulated particle trajectories in a surface of a section of the Hamiltonian (2). (a) Modulation tune 0.000 404, (b) modulation tune 0.000472 , (c) modulation tune 0.000499 , and (d) modulation tune 0.000553 . All cases are driven with a modulation amplitude of $\epsilon=0.8$.
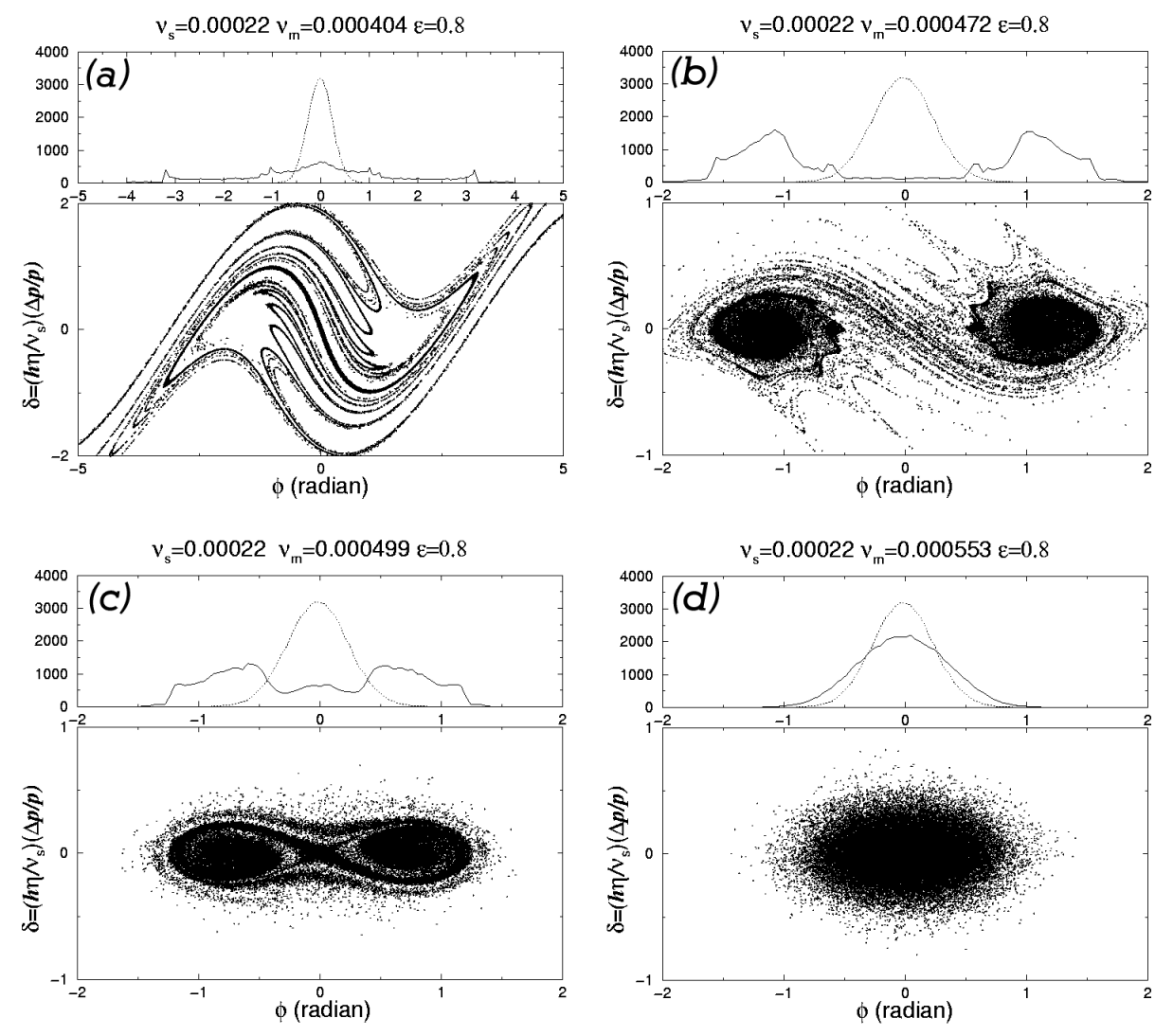

FIG. 4. Tracking simulations starting with 50000 particles in a Gaussian distribution and using the same parameters as in Fig. 3. In all cases the parameter $\epsilon$ is ramped from 0 to 0.8 in ten synchrotron periods. 

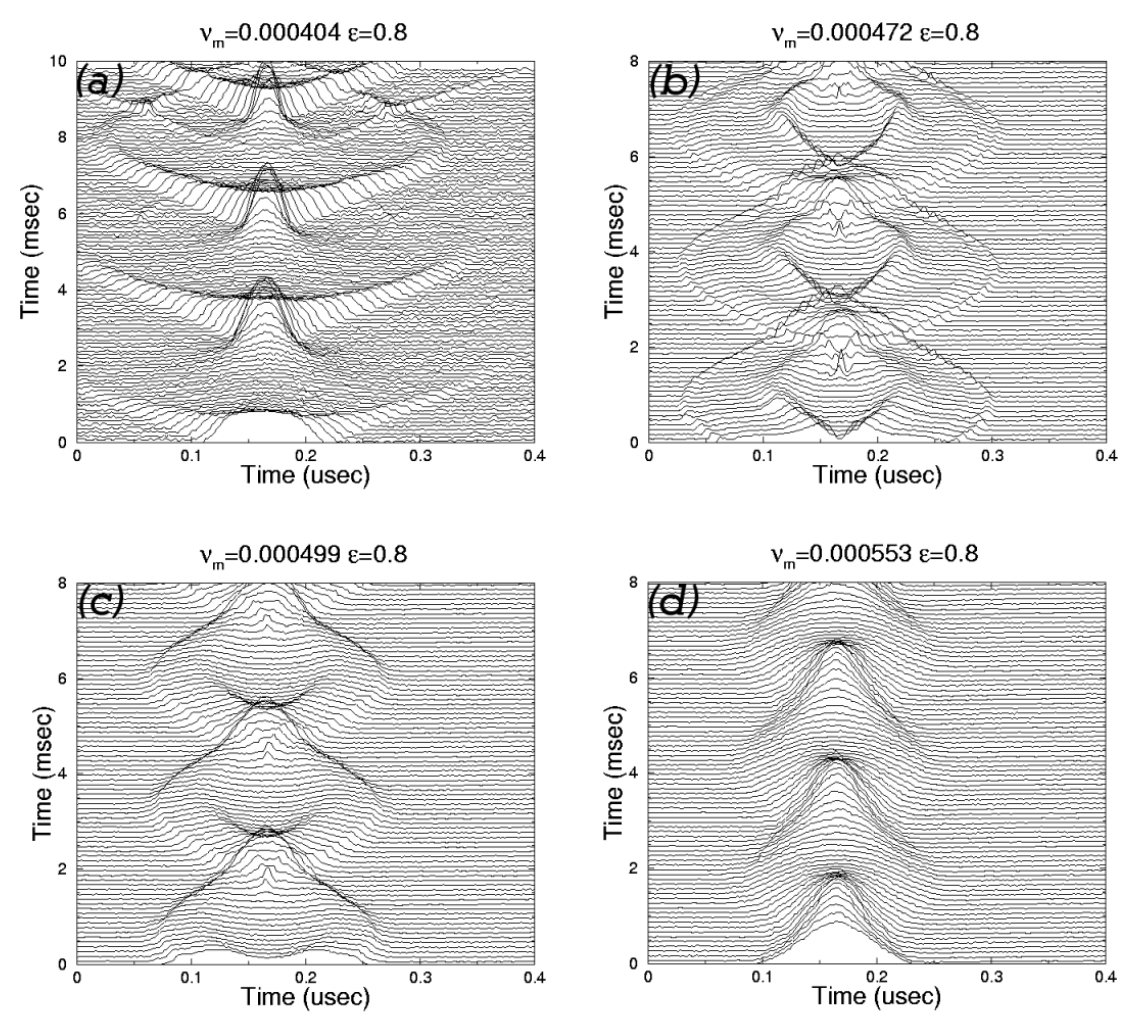

FIG. 5. Measurements of driven bunch shape oscillations for different modulation frequencies, as seen on a wall current monitor. (a) Modulation tune 0.000404 , (b) modulation tune 0.000472 , (c) modulation tune 0.000499 , and (d) modulation tune 0.000553. In all cases the parameter $\epsilon$ is ramped from 0 to 0.8 in ten synchrotron periods.
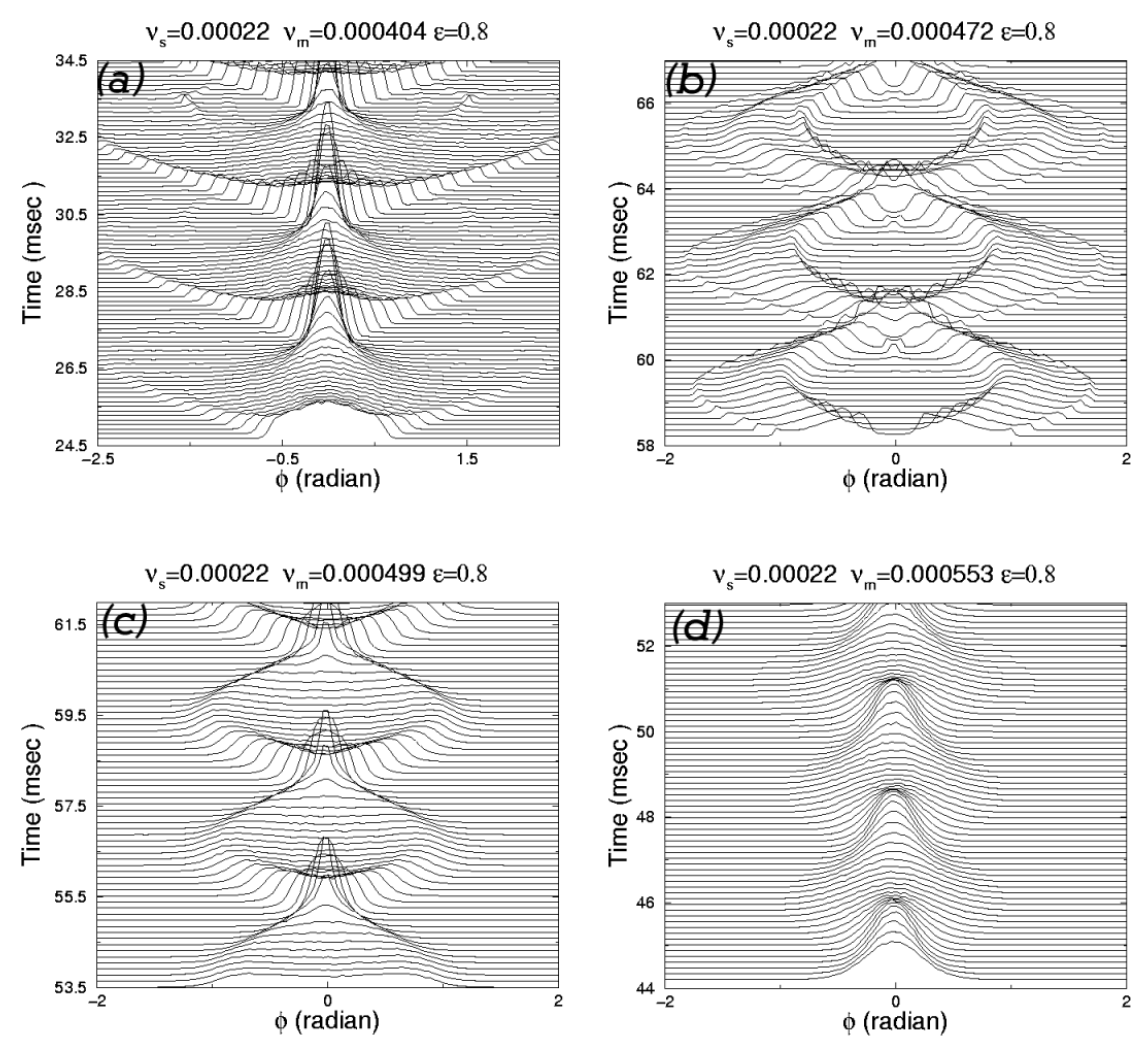

FIG. 6. Simulated mountain range results of tracking 50000 particles for each of the cases shown in Figs. 3-5. 
region of phase space than used for Figs. 3(b)-3(d) and 4(b)-4(d). In Figs. 3(b)-3(d) and 4(b)-4(d) we show the phase space well after reaching full modulation amplitude. In Fig. 4 each figure shows a profile of the initial distribution as well as a profile of the final distribution.

Figure 5 shows the measured mountain range plots for these four cases. Figure 6 shows simulated mountain ranges, using 50000 particles, for each of the above cases. These simulation results agree very well with the measured mountain range plots shown in Fig. 5. We attribute the small remaining discrepancies to a mismatch in the initial distributions.

\section{SUMMARY}

Our simulations of adiabatic excitation of longitudinal parametric resonances match experimentally obtained data extremely well over different parameter regimes. Thus the one-turn map in Eq. (1) that is used in the simulation and includes only single particle effects is an accurate model for the parameter regimes of our experiments.

\section{ACKNOWLEDGMENTS}

This work was supported by the U.S. Department of Energy. The authors wish to acknowledge M. Blaskiewicz, J.M. Brennan, and T. Satogata at BNL, each of whom offered very helpful discussions.

\section{APPENDIX: SIMULATOR CODE DETAILS}

The simulator is composed of fewer than 500 lines of $\mathrm{C}++$ code and was developed and debugged very quickly. Individual particles are tracked for many revolutions using a set of nested loops. First, we define the particle distribution in phase space, then, for each particle, we track over a given number of modulation periods and for the number of revolutions per modulation period. We can either print out particle coordinates in phase space every modulation period to get the surface of sections of the Hamiltonian (2) or just print out the final coordinates after the given total number of revolutions. We can generate a Gaussian or uniform beam distribution. We also include the code for generating mountain range plots, for direct comparison to actual data. The simulator used well-known techniques and algorithms. For example, to generate a Gaussian distribution we did the following:

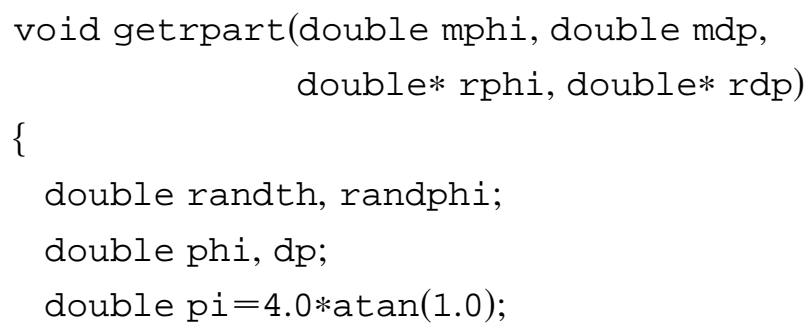

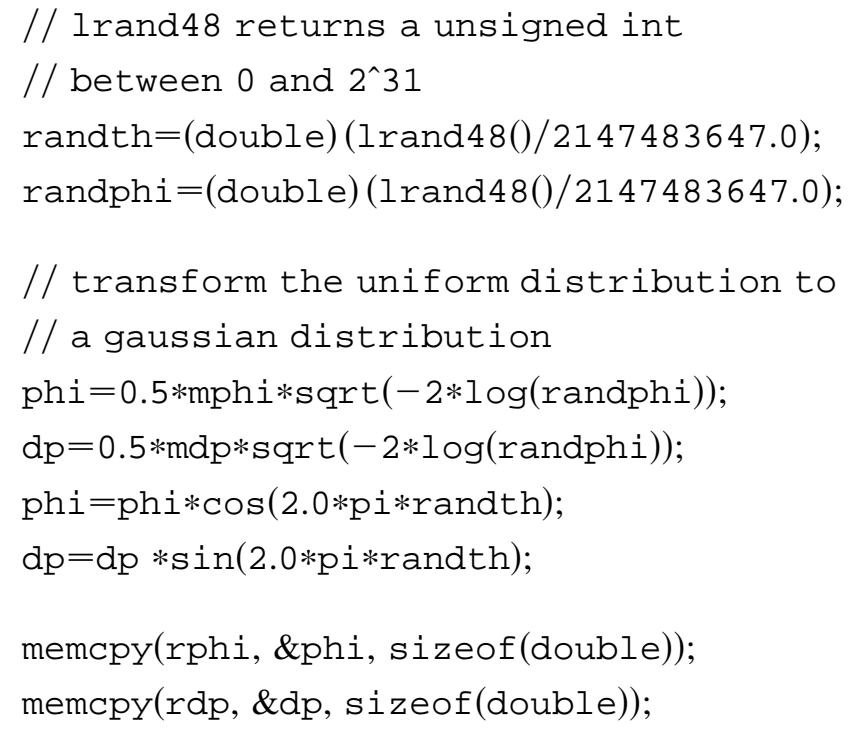

The application does not generate any graphics, but simply dumps results into files. We use the xmgr (ACE/gr [10]) plotting package to display our results. The user interface is a file input that specifies the synchrotron tune, the modulation tune, the modulation amplitude, the number of revolutions per modulation period, the number of modulation periods to sample over, and the number of revolutions to ramp the amplitude. Also specified are various other parameters to control which distribution to use (including a random seed value for generating the Gaussian distribution) and to control the mountain range output.

[1] M. Bai et al., Phys. Rev. ST Accel. Beams 3, 064001 (2000).

[2] D. Li et al., Nucl. Instrum. Methods Phys. Res., Sect. A 364, 205-223 (1995).

[3] D. A. Edwards and M. J. Syphers, An Introduction to the Physics of High Energy Accelerators (Wiley, New York, 1993).

[4] D. Li et al., Phys. Rev. E 48, 1638-1641 (1993).

[5] J. M. Brennan, in Proceedings of the 1995 Particle Accelerator Conference, Dallas, Texas (IEEE, Piscataway, NJ, 1996), Vol. 3, pp. 1489-1493.

[6] J. M. Brennan (private communication).

[7] J. M. Brennan (private communication).

[8] B. Zotter, in Handbook of Accelerator Physics and Engineering, edited by A. Chao and M. Tigner (World Scientific, Singapore, 1999).

[9] M. Blaskiewicz (private communication).

[10] http://plasma-gate.weizmann.ac.il/Xmgr/ 